Discrete Comput Geom 33:549-563 (2005)

DOI: $10.1007 /$ s00454-004-1124-4

\title{
Generic Global Rigidity
}

\author{
Robert Connelly* \\ Department of Mathematics, \\ Malott Hall, Cornell University, \\ Ithaca, NY 14853, USA \\ connelly@math.cornell.edu
}

\begin{abstract}
Suppose a finite configuration of labeled points $\mathbf{p}=\left(p_{1}, \ldots, p_{n}\right)$ in $\mathbb{E}^{d}$ is given along with certain pairs of those points determined by a graph $G$ such that the coordinates of the points of $\mathbf{p}$ are generic, i.e., algebraically independent over the integers. If another corresponding configuration $\mathbf{q}=\left(q_{1}, \ldots, q_{n}\right)$ in $\mathbb{E}^{d}$ is given such that the corresponding edges of $G$ for $\mathbf{p}$ and $\mathbf{q}$ have the same length, we provide a sufficient condition to ensure that $\mathbf{p}$ and $\mathbf{q}$ are congruent in $\mathbb{E}^{d}$. This condition, together with recent results of Jackson and Jordán [JJ], give necessary and sufficient conditions for a graph being generically globally rigid in the plane.
\end{abstract}

\section{Introduction}

A fundamental problem in distance geometry is to determine when the distances between certain pairs of vertices of a finite configuration in Euclidean space $\mathbb{E}^{d}$ determine it up to congruence. To put this more precisely, we use the language from the rigidity of bar frameworks.

\subsection{Global rigidity}

A configuration is a finite collection of $n$ labeled points, $\mathbf{p}=\left(p_{1}, \ldots, p_{n}\right)$, where each $p_{i} \in \mathbb{E}^{d}$, for $1 \leq i \leq n$. We say the configuration $\mathbf{p}$ is in $\mathbb{E}^{d}$. A graph $G$ will always be finite, undirected, and without loops or multiple edges. A bar framework in $\mathbb{E}^{d}$ is a graph $G$ with $n$ vertices together with a corresponding configuration $\mathbf{p}=\left(p_{1}, \ldots, p_{n}\right)$ in $\mathbb{E}^{d}$, and is denoted by $G(\mathbf{p})$. We represent a framework graphically as in Fig. 1, where in this case $G$ is $K_{4}$, the complete graph on four vertices. See [AR1] and [Ar2] for these basic definitions and background.

* This research was supported in part by NSF Grant No. DMS-0209595. 


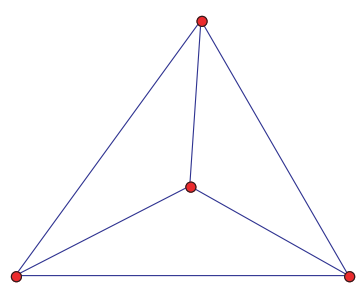

Fig. 1. The complete graph $K_{4}$.

Here the vertices are represented as small circular points, and line segments, which represent bars, may cross without a vertex at the intersection.

We say that two frameworks $G(\mathbf{p})$ and $G(\mathbf{q})$ are equivalent, and we write $G(\mathbf{p}) \equiv$ $G(\mathbf{q})$ if when $\{i, j\}$ forms an edge of $G$, then $\left|p_{i}-p_{j}\right|=\left|q_{i}-q_{j}\right|$. We say that a configuration $\mathbf{p}=\left(p_{1}, \ldots, p_{n}\right)$ is congruent to $\mathbf{q}=\left(q_{1}, \ldots, q_{n}\right)$, and we write $\mathbf{p} \equiv \mathbf{q}$ if for all $\{i, j\}$ in $\{1, \ldots, n\},\left|p_{i}-p_{j}\right|=\left|q_{i}-q_{j}\right|$. A framework $G(\mathbf{p})$ is called globally rigid in $\mathbb{E}^{d}$ if $G(\mathbf{p}) \equiv G(\mathbf{q})$ implies $\mathbf{p} \equiv \mathbf{q}$. In the past we have used the term "uniquely realized" for globally rigid, and this is the term that is used in [JJ], where the term globally rigid is reserved for generic global rigidity for a graph $G$. (A definition of generic global rigidity will be given shortly.) We stay with the definitions here.

Figure 2 shows some examples of frameworks that are globally rigid in the plane. For the example on the left, if the vertices are perturbed a sufficiently small amount, it will remain globally rigid in the plane, whereas that is not the case for the other two frameworks. The middle vertex in the middle figure must lie along the straight line determined by the other two adjacent vertices. The six vertices on the figure on the right must lie on a conic (in the order shown) to ensure global rigidity.

Figure 3 shows some examples that are not globally rigid in the plane. The example on the left is not rigid in the plane, whereas the other three are rigid in the plane. The middle two examples have non-equivalent realizations obtained by reflecting one of the vertices about a line. (The third configuration is chosen such that the appropriate three vertices are collinear, making it non-generic.) The example on the right is not globally rigid, even though its configuration is generic, by a theorem from [H3].

A framework $G(\mathbf{p})$ in $\mathbb{E}^{d}$ is said to be rigid if there is an $\varepsilon>0$ such that for any other configuration $\mathbf{q}$ in $\mathbb{E}^{d}$, where $|\mathbf{p}-\mathbf{q}|<\varepsilon$ and $G(\mathbf{p}) \equiv G(\mathbf{q})$, then $\mathbf{p} \equiv \mathbf{q}$. All frameworks in Figs. 1 and 2 and all but the left framework in Fig. 3 are rigid in the plane.
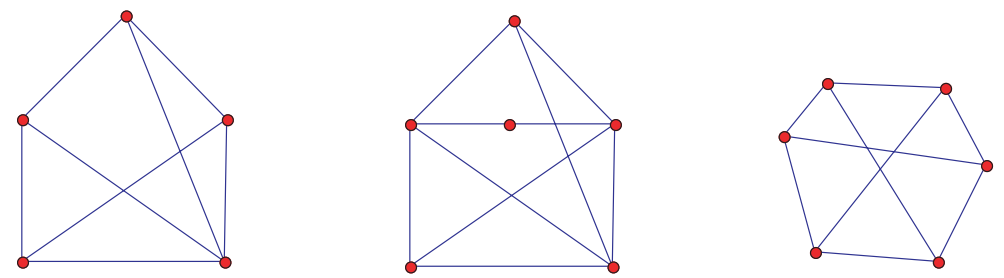

Fig. 2. Globally rigid frameworks in the plane. 

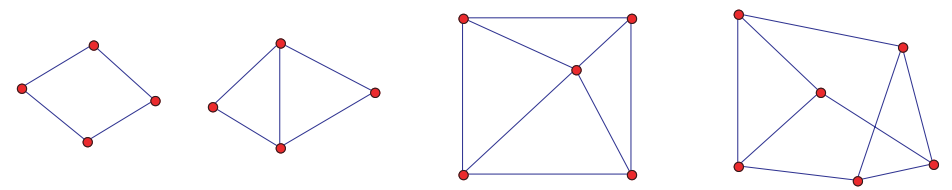

Fig. 3. Frameworks not globally rigid in the plane.

In [C1] and [C3] there are techniques for showing that some frameworks are globally rigid, essentially using tensegrity frameworks. (Tensegrity frameworks are similar to the bar frameworks which we have defined here, but they involve inequalities replacing equality distance constraints in the definition.)

One can ask, for a given bar framework $G(\mathbf{p})$, whether it is globally rigid. However, in $[\mathrm{Sa}]$ it is shown that this problem is strongly NP hard even for bar frameworks in $\mathbb{E}^{1}$. We will show that there is an algebraic set of configurations, defined by polynomial equations in the coordinates of the configuration, such that when the configuration $\mathbf{p}$ is outside that set, $G(\mathbf{p})$ is globally rigid in $\mathbb{E}^{2}$. However, the complexity of that set of configurations appears to be exponential in $n$, the number of points of the configuration.

\subsection{Generic Global Rigidity}

So, we are led to consider the question of whether "most" configurations $\mathbf{p}$ for a given graph $G$ are globally rigid. A set $A=\left(\alpha_{1}, \ldots, \alpha_{m}\right)$ of distinct real numbers is said to be algebraically dependent if there is a non-zero polynomial $h\left(x_{1}, \ldots, x_{m}\right)$ with integer coefficients such that $h\left(\alpha_{1}, \ldots, \alpha_{m}\right)=0$. If $A$ is not algebraically dependent, it is called generic. If a configuration $\mathbf{p}=\left(p_{1}, \ldots, p_{n}\right)$ in $\mathbb{E}^{d}$ is such that its $d n$ coordinates are generic, we say $\mathbf{p}$ is generic.

We raise a possibly more tractable problem. For a given graph $G$, when $G(\mathbf{p})$ is globally rigid for all generic configurations $\mathbf{p}$ in $\mathbb{E}^{d}$ we say that $G$ itself is generically globally rigid in $\mathbb{E}^{d}$. So for a fixed dimension $d$ we ask whether a given graph $G$ is generically globally rigid. For $d=1$, it is easy to see that $G$ is generically globally rigid if and only if $G$ is vertex 2-connected, which means that it takes the removal of at least two vertices of $G$ to disconnect the rest of the vertices. In general a graph is vertex $m$-connected if it takes the removal of at least $m$ vertices of $G$ to disconnect the rest of the vertices.

For $d=2$, by combining the results of [H3], [JJ], and here we now have complete information about generic global rigidity for any graph. We first describe the result in [H3]. A framework $G(\mathbf{p})$ in $\mathbb{E}^{d}$ is said to be redundantly rigid if $G(\mathbf{p})$ is rigid in $\mathbb{E}^{d}$ even after the removal of any edge of $G$. The following is a main result in [H3].

Theorem 1.1 [H3]. Let $G(\mathbf{p})$ be a framework in $\mathbb{E}^{d}$ such that the configuration $\mathbf{p}$ is generic, and $G(\mathbf{p})$ is globally rigid with at least $d+1$ vertices. Then the following conditions must hold:

(i) The graph $G$ is vertex $(d+1)$-connected.

(ii) The framework $G(\mathbf{p})$ is redundantly rigid in $\mathbb{E}^{d}$. 
Condition (i) is clear. One just reflects the vertices on one side of a hyperplane through any separating set of $d$ vertices as in the leftmost two frameworks of Fig. 3. Condition (ii) is more subtle. Roughly the idea is to remove an edge from $G$, let the resulting framework flex, and replace the edge in a different configuration. It is natural to conjecture that conditions (i) and (ii) are sufficient for generic global rigidity as well as necessary. Unfortunately, for $d \geq 3$, in [C2], that conjecture is shown to be false. For $d=3$, the complete bipartite graph $K(5,5)$ is redundantly rigid, vertex 4-connected, but there are generic configurations, where the corresponding framework is not globally rigid, and it is the only known example.

For $d=2$, thanks to a recent result of Jackson and Jordán [JJ, Theorem 1.1], combined with the result here, Theorem 1.3, conditions (i) and (ii) give a complete description of when a graph $G$ is generically globally rigid. See also [BJ] for the result when case $G$ has $2 n-2$ edges, the minimum possible for generic global rigidity in the plane when $G$ has $n$ vertices. (See page 99 of [GSS] for a statment of my conjecture when $G$ has $2 n-2$ edges, as well as [H2] )

For $d \geq 3$ it is somewhat embarrassing to admit that it is not known whether global rigidity is a generic property. This means that if $G(\mathbf{p})$ is a generically rigid framework in $\mathbb{E}^{d}$, and $\mathbf{q}$ is another generic configuration in $\mathbb{E}^{d}$, it is not known, except for $d=1$ or $d=2$, whether $G(\mathbf{q})$ is globally rigid. This question was first pointed out by Maria Terrell.

On the other hand, it is known that rigidity is a generic property. In other words, if $\mathbf{p}$ is a generic configuration in $\mathbb{E}^{d}$, and $\mathbf{q}$ is another generic configuration in $\mathbb{E}^{d}$, it is known that $G(\mathbf{q})$ is rigid if and only if $G(\mathbf{p})$ is rigid. This is discussed in [G] and [AR1], for example. Thus rigidity in $\mathbb{E}^{d}$ is entirely a combinatorial property of the graph $G$, although a purely combinatorial polynomial time algorithm to determine generic rigidity is known only for $d=1$ and $d=2$. The result here and in [JJ] verifies the correctness of the polynomial time algorithm in [H3]. This algorithm determines generic redundant rigidity in $\mathbb{E}^{2}$ and vertex 3-connectivity of a graph $G$ in deterministic polynomial time, and thus generic global rigidity in $\mathbb{E}^{2}$.

\subsection{Stresses and Stress Matrices}

In order to state the main result here, we need to define the notion of an equilibrium stress. Suppose that $G$ is a graph with $n$ vertices. Any set of scalars $\omega_{i j}=\omega_{j i}$ defined for all pairs of vertices for all $\{i, j\}$ in $\{1, \ldots, n\}$, such that $\omega_{i j}=0$ when $\{i, j\}$ is not an edge of $G$, is called a stress for $G$. We combine these scalars into one row vector $\omega=\left(\ldots, \omega_{i j}, \ldots\right)$, where there is one and only one coordinate in $\omega$ for each edge $\{i, j\}$ of $G$, where $i \neq j$. We regard $\omega=\left(\ldots, \omega_{i j}, \ldots\right)$ as a stress for $G$.

If $\omega=\left(\ldots, \omega_{i j}, \ldots\right)$ is a stress for a graph $G$, we say that it is an equilibrium stress for the framework $G(\mathbf{p})$ if, for each vertex $i$ of $G$, the following equilibrium vector equation holds:

$$
\sum_{j} \omega_{i j}\left(p_{j}-p_{i}\right)=0 .
$$

When $\omega$ is an equilibrium stress for the framework $G(\mathbf{p})$, the graph $G$ does not appear explicitly in (1) since the non-edges of $G$ have zero stress. So we can test $\omega$ for 
equilibrium for any other configuration $q$ suppressing the reference to the graph $G$.

To each stress for a graph $G$ on $n$ vertices, there is an $n$-by- $n$ symmetric matrix $\Omega$, the associated stress matrix, such that for $i \neq j,\{i, j\}$ in $\{1, \ldots, n\}$, the $i j$ entry of $\Omega$ is $-\omega_{i j}$, and the diagonal entries are such that the row and column sums of the entries of $\Omega$ are zero. Recall that the affine span of a configuration of points in $\mathbb{E}^{d}$ is the smallest affine subspace of $\mathbb{E}^{d}$ that contains the points, and that an affine image of a configuration $\mathbf{p}=\left(p_{1}, \ldots, p_{n}\right)$ is $\left(\alpha\left(p_{1}\right), \ldots, \alpha\left(p_{n}\right)\right)$, where $\alpha$ is an affine linear map $\alpha: \mathbb{E}^{d} \rightarrow \mathbb{E}^{d}$. The following are some of the basic properties of stress matrices and can be found in [C1].

Proposition 1.2. If $\omega=\left(\ldots, \omega_{i j}, \ldots\right)$ is an equilibrium stress for the framework $G(\mathbf{p})$ in $\mathbb{E}^{d}$, and the affine span of $\mathbf{p}$ is all of $\mathbb{E}^{d}$, then the following hold:

(i) The rank of the associated stress matrix $\Omega$ is at most $n-d-1$.

(ii) If the rank of $\Omega$ is $n-d-1$ and $\omega$ is an equilibrium stress for any other framework $G(\mathbf{q})$, then the configuration $\mathbf{q}$ is an affine image of the configuration $\mathbf{p}$.

If an equilibrium stress $\omega$ for the configuration $\mathbf{p}$ satisfies condition (ii) above, such that its stress matrix $\Omega$ has rank $n-d-1$, we say that the configuration $\mathbf{p}$ is universal with respect to $\omega$.

\subsection{The Main Result}

We are now in a position to state our main result.

Theorem 1.3. Suppose that $\mathbf{p}=\left(p_{1}, \ldots, p_{n}\right)$ is a generic configuration in $\mathbb{E}^{d}$ such that there is an equilibrium stress $\omega$ for a framework $G(\mathbf{p})$, where the rank of the associated stress matrix $\Omega$ is $n-d-1$. Then $G(\mathbf{p})$ is globally rigid in $\mathbb{E}^{d}$.

The proof of this result occupies most of the later sections of this paper. Note that if we have a generic configuration $\mathbf{p}$, it is possible to solve the equilibrium equation (1) for an appropriate equilibrium stress $\omega$, and then calculate the rank of $\Omega$. If the rank is maximal as in Theorem 1.3 , we can be assured that $G(\mathbf{p})$ is globally rigid in $\mathbb{E}^{d}$. By choosing a random configuration, solving the equilibrium equations numerically with appropriate estimates for the accuracy, and calculating the rank of $\Omega$ using those estimates, we get an algorithm that detects global rigidity with high probablity, assuming that the hypothesis of Theorem 1.3 holds for the graph $G$ in question. (Here random can mean choosing a configuration with a uniform distribution in a bounded neighborhood of any given configuration. The set of non-generic configurations in the space of all configurations is of measure 0 , as pointed out in [G].) This leads us to the following conjecture, essentially a converse to the result above. A simplex is a framework $G(\mathbf{p})$ in $\mathbb{E}^{d}$, where $G$ is the complete graph (i.e., all pairs of vertices form an edge of $G$ ), and the vertices of $\mathbf{p}=\left(p_{1}, \ldots, p_{n}\right)$ are affine independent. (So in particular $n \leq d+1$.)

Conjecture 1.4. Suppose that $\mathbf{p}=\left(p_{1}, \ldots, p_{n}\right)$ is a generic configuration in $\mathbb{E}^{d}$ such that $G(\mathbf{p})$ is globally rigid in $\mathbb{E}^{d}$. Then either $G(p)$ is a simplex or there is a non- 
zero equilibrium stress $\omega$ for $G(\mathbf{p})$, where the rank of the associated stress matrix $\Omega$ is $n-d-1$.

It is easy to verify this conjecture for $d=1$, and in light of [JJ] the conjecture is now known for $d=2$.

It should also be pointed out that the rank of $\Omega$ alone is not enough to ensure global rigidity. For example, the framework of Fig. 3 which has five vertices has a stress matrix of maximal rank, even though its central vertex is on the diagonal of the square. Nevertheless, it is not globally rigid in the plane. As mentioned earlier, the equations that describe global rigidity might be quite complicated.

\subsection{Consequences}

Theorem 1.3 can be used to provide purely combinatorial conditions for generic global rigidity, and give a complete description for generic global rigidity in the plane. First we describe a geometric construction for frameworks.

Suppose that $G(\mathbf{p})$ is a framework in $\mathbb{E}^{d}$, and $\{i, j\}$ is an edge of $G$ such that $p_{i} \neq p_{j}$. Remove the edge $\{i, j\}$ from $G$ and replace it with $d+1$ others, all connected to a new vertex $p_{k}$, which lies on the line through $p_{i}$ and $p_{j}$ (but it is not equal to $p_{i}$ and $p_{j}$ ), and which in turn is connected to $p_{i}, p_{j}$, and some other set of $d-1$ distinct vertices besides $p_{i}$ and $p_{j}$. Call the new framework $\sigma G(\sigma \mathbf{p})$, and this operation a Hennenberg operation on $G(\mathbf{p})$. Denote the new graph by $\sigma G$, and the new configuration by $\sigma \mathbf{p}$. We also say that $\sigma G$ is obtained from $G$ by a Hennenberg operation. (Note that there are other Hennenberg operations on a graph, but this is the only one that we consider in this paper. For example, see page 134 of [GSS] for a discussion of this, as well as [TW].) See Fig. 4 for an example.

Theorem 1.5. Suppose that $\mathbf{p}=\left(p_{1}, \ldots, p_{n}\right)$ is a generic configuration in $\mathbb{E}^{d}$, and $\sigma G$ is obtained from a graph $G$ by a Hennenberg operation such that the following hold:

(i) There is a non-zero equilibrium stress $\omega$ for the framework $G(\mathbf{p})$, where the rank of the associated stress matrix $\Omega$ is $n-d-1$.

(ii) $G(\mathbf{p})$ is rigid in $\mathbb{E}^{d}$.

If $\mathbf{q}$ is another generic configuration of $n+1$ vertices in $\mathbb{E}^{d}$, then (i) and (ii) hold for $(\sigma G)(\mathbf{q})$ as well, with $n$ replaced by $n+1$.

This theorem can now be applied to the following theorem by Jackson and Jordán [JJ].

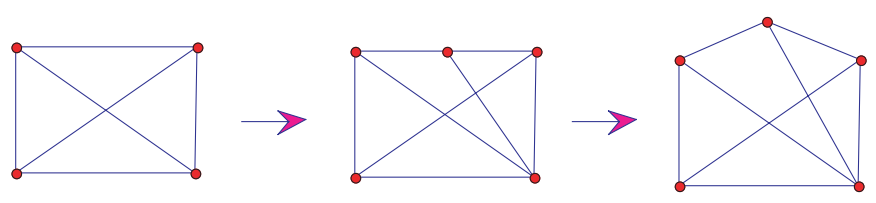

Fig. 4. The Hennenberg operation. 
Theorem 1.6 [JJ]. If $G$ is generically redundantly rigid in $\mathbb{E}^{2}$, and vertex 3 -connected, then $G$ can be obtained from $K_{4}$ by a sequence of Hennenberg operations and edge insertions.

Hence we get the following result, which was a conjecture of Hendrickson:

Corollary 1.7. A graph $G$ is generically globally rigid in $\mathbb{E}^{2}$ if and only if $G$ is generically redundantly rigid in $\mathbb{E}^{2}$, and vertex 3 -connected, or $G$ is a complete graph on fewer than four vertices.

Proof. The "only if" part of Corollary 1.7 is Theorem 1.1 of [H3]. The "if" part was the conjecture, and it follows inductively, starting with $K_{4}$, by applying Theorem 1.6 obtaining $G$ after a sequence of Hennenberg operations. Theorem 1.5 assures that the rank of a stress matrix is maximal at each stage for a generic configuration. Then finally apply Theorem 1.3 to conclude that $G(\mathbf{p})$ is globally rigid for a generic configuration $\mathbf{p}$.

Both redundant rigidity in $\mathbb{E}^{2}$ and 3-connectivity can be checked deterministically in polynomial time in $n$, the number of vertices of $G$.

In the following we present the proof of the main result, Theorem 1.3, as well as Theorem 1.5.

\section{The Rigidity Map and the Rigidity Matrix}

We review some rigidity theory that we need. Suppose that $G(\mathbf{p})$ is a framework with $n$ vertices and $e$ edges in $\mathbb{E}^{d}$. Let

$$
f: \mathbb{E}^{\text {nd }} \rightarrow \mathbb{E}^{e}
$$

be the rigidity map defined by $f(\mathbf{p})=\left(\ldots,\left|p_{i}-p_{j}\right|^{2}, \ldots\right)$. This is the map that takes the space of configurations to the space of metrics, or more accurately the space of squared edge lengths. Note that $f$ is an integral polynomial function, and that the differential of $f$ is given by

$$
d f_{\mathbf{p}}\left(\mathbf{p}^{\prime}\right)=2\left(\ldots,\left(p_{i}-p_{j}\right) \cdot\left(p_{i}^{\prime}-p_{j}^{\prime}\right), \ldots\right) .
$$

It is helpful to consider the matrix of $d f$ with respect to the standard basis. With this in mind, we define the rigidity matrix as

$$
R(\mathbf{p})=\left[\begin{array}{llllllll} 
& & & & & \\
0 & \cdots & \left(p_{i}-p_{j}\right) & \cdots & 0 & \cdots\left(p_{j}-p_{i}\right) & \cdots & 0
\end{array}\right] .
$$

The columns of $R(\mathbf{p})$ are regarded as $n$ sets of $d$ columns, where each set of $d$ columns corresponds to the vertices of $G$. The rows of $R(\mathbf{p})$ correspond to the edges of $G$, and the entries of each row are all zero, except for the two groups of $d$ coordinates corresponding to the vertices adjacent to the given edge. It is easy to check that $d f_{\mathbf{p}}\left(\mathbf{p}^{\prime}\right)=2 R(\mathbf{p}) \mathbf{p}^{\prime}$, 
where we regard $\mathbf{p}^{\prime}=\left(p_{1}^{\prime}, \ldots, p_{n}^{\prime}\right)$ as an $n d$ column vector. In fact, we can also regard $\mathbf{p}^{\prime}$ as a configuration of $n$ vectors in $\mathbb{E}^{d}$. We say that $\mathbf{p}^{\prime}$ is an infinitesimal flex of the framework $G(\mathbf{p})$ in $\mathbb{E}^{d}$ if $R(\mathbf{p}) \mathbf{p}^{\prime}=0$, and that $\mathbf{p}^{\prime}$ is a trivial infinitesimal flex if there is a differentiable family of congruences of $\mathbb{E}^{d}$ starting at the identity, such that each $p_{i}^{\prime}$ is the derivative restricted to $p_{i}$ at time 0 . See [CW], for example, for more details. With this in mind, we say that a framework $G(\mathbf{p})$ in $\mathbb{E}^{d}$ is infinitesimally rigid if the only infinitesimal flexes of $G(\mathbf{p})$ are trivial. A basic result is the following.

Theorem 2.1. If $G(\mathbf{p})$ is infinitesimally rigid in $\mathbb{E}^{d}$, then it is rigid in $\mathbb{E}^{d}$.

A proof can be found in [G], [AR1], or [CW], for example. When the affine span of the configuration $\mathbf{p}=\left(p_{1}, \ldots, p_{n}\right)$ is all of $\mathbb{E}^{d}$, the trivial infinitesimal flexes of $\mathbf{p}$ form a linear subspace of dimension $d(d+1) / 2$. This leads to the following, which can also be found in the references above.

Proposition 2.2. A framework $G(\mathbf{p})$ is infinitesimally rigid in $\mathbb{E}^{d}$ if and only if either the rank of $R(\mathbf{p})$ is $n d-d(d+1) / 2$ or $G(\mathbf{p})$ is a simplex.

Proposition 2.3. Suppose that $G(\mathbf{p})$ is a framework in $\mathbb{E}^{d}$, and $\sigma G$ is obtained from a graph $G$ by a Hennenberg operation, where the $d-1$ additional edges and the subdivided edge of $G$ do not lie in a $(d-1)$-dimensional affine plane. Then $(\sigma G)(\sigma \mathbf{p})$ is infinitesimally rigid.

A proof of this can be found in [TW].

For a generic configuration $\mathbf{p}$ in $\mathbb{E}^{d}$, the rank of $R(\mathbf{p})$ is constant in a sufficiently small neighborhood of $\mathbf{p}$, since it is determined by the determinant of appropriately choosen minors.

Corollary 2.4. A framework $G(\mathbf{p})$ is rigid for $\mathbf{p}$, a generic configuration in $\mathbb{E}^{d}$, if and only if either the rank of $R(\mathbf{p})$ is $n d-d(d+1) / 2$ or $G(\mathbf{p})$ is a simplex.

A good source for the proof of this, which uses the inverse function theorem, is again [AR1] as well as [G].

Equilibrium stresses can also be described in terms of the rigidity matrix. We regard a stress $\omega=\left(\ldots, \omega_{i j}, \ldots\right)$ as a row vector. The following is a straightforward calculation:

Lemma 2.5. A stress $\omega=\left(\ldots, \omega_{i j}, \ldots\right)$ for a framework $G(\mathbf{p})$ is an equilibrium stress if and only if $\omega R(\mathbf{p})=0$.

In other words, $\omega$ is an element of the cokernel of the rigidity matrix $R(\mathbf{p})$.

\section{Generic Facts}

Here we record some properties that we will need for generic configurations with regard to rigidity properties. 
Proposition 3.1. Suppose that $f: \mathbb{E}^{a} \rightarrow \mathbb{E}^{b}$ is a function, where each coordinate is a polynomial with integer coefficients, and $\mathbf{p} \in \mathbb{E}^{a}$ is generic. Then the differential at $\mathbf{p}$, $d f_{\mathbf{p}}$, has maximum rank.

Proof. The rank of $d f_{\mathbf{p}}$ is the largest $m$ such that the rank of a square $m$-by- $m$ minor is $m$. So those configurations $\mathbf{q}$, where the determinant of all the $m$-by- $m$ minors of $d f_{\mathbf{q}}$ are zero are not generic configurations, since their coordinates satisfy a non-trivial polynomial equation with integer entries.

When it comes to the rigidity of a framework $G(\mathbf{p})$, Proposition 3.1 essentially says that generic points are all the same as far as rigidity goes.

Suppose that $X \subset \mathbb{E}^{a}$. We say $X$ is an integral semi-algebraic set or just a semialgebraic set if it is given by a system of polynomial equations and inequalities over the integers. The following is useful. The proof can be found in [Ma], for example. The general theory works in case the coefficients of the polynomials are more general than the case when they are the real integers, as the case here, but this is enough for our needs.

Lemma 3.2. Finite intersections, finite unions, images, and inverse images under integral polynomial functions of semi-algebraic sets are semi-algebraic sets. In particular, any point in a semi-algebraic set of dimension less than a in $\mathbb{E}^{a}$ is not a generic point.

We come to one of the crucial observations of this paper.

Proposition 3.3. Suppose that $f: \mathbb{E}^{a} \rightarrow \mathbb{E}^{b}$ is a function, where each coordinate is a polynomial with integer coefficients, $\mathbf{p} \in \mathbb{E}^{a}$ is generic, and $f(\mathbf{p})=f(\mathbf{q})$, for some $\mathbf{q} \in$ $\mathbb{E}^{a}$. Then there are (open) neighborhoods $N_{\mathbf{p}}$ of $\mathbf{p}$ and $N_{\mathbf{q}}$ of $\mathbf{q}$ in $\mathbb{E}^{a}$ and a diffeomorphism $g: N_{\mathbf{q}} \rightarrow N_{\mathbf{p}}$ such that for all $x \in N_{\mathbf{q}}, f(g(x))=f(x)$, and $g(\mathbf{q})=\mathbf{p}$.

Proof. We assume, without loss of generality, that $\mathbf{p} \neq \mathbf{q}$. Suppose the maximum rank of the differential of $f$ is $m$. By Proposition 3.1 we know that the rank of the differential of $f$ at $\mathbf{p}, d f_{\mathbf{p}}$, is $m$, and there must be a neighborhood of $p, U_{\mathbf{p}}$, where $\operatorname{dim} d f_{\mathbf{x}}=m$ for all $x \in U_{\mathbf{p}}$. By the inverse function theorem we can restrict $U_{\mathbf{p}}$ such that $f\left(U_{\mathbf{p}}\right)$ is diffeomorphic to $\mathbb{E}^{m}$.

Consider the set

$$
T=\left\{x \in \mathbb{E}^{a} \mid \exists y \in \mathbb{E}^{a}, f(x)=f(y) \wedge \operatorname{rank}\left[d f_{y}\right]<m\right\} .
$$

By Sard's theorem (see [Mi] for a good presentation), we know that $\mathbb{E}^{a}-T$ is dense in $\mathbb{E}^{a}$. Since $T$ contains no open set, it must be of dimension strictly less than $m$. Since the point $\mathbf{p}$ is generic, by Lemma 3.2, $\mathbf{p} \notin T$.

Thus $f(\mathbf{p})=f(\mathbf{q})$ is a regular value, i.e., the image only of points whose differential has maximal rank, since $T$ is another semi-algebraic set of dimension less than $a$. So $f$ is a submersion at the points $\mathbf{p}$ into $f\left(U_{p}\right)$ and $\mathbf{q}$ into $f\left(U_{p}\right)$.

Next we wish to be assured that a neighborhood of $\mathbf{q}$ can be chosen so that its image is also $f\left(U_{\mathbf{p}}\right)$. To this end we consider those points $S$ of $\mathbb{E}^{a}$ which have neighborhoods that map diffeomorphically onto an open subset of $f\left(\mathbb{E}^{a}\right)$. For any $\varepsilon>0$ and $x \in \mathbb{E}^{a}$ 
let $U_{x}(\varepsilon)=\left\{y \in \mathbb{E}^{a}|| y-\left.x\right|^{2}<\varepsilon\right\}$ and the usual set notation, $f\left(U_{x}(\varepsilon)\right)=\left\{z \in \mathbb{E}^{b} \mid\right.$ $\exists y \in U_{x}(\varepsilon) \wedge f(y)=z$. By Lemma 3.2, $U_{x}(\varepsilon), f\left(U_{x}(\varepsilon)\right)$, etc., are semi-algebraic sets. With this is mind, define the following:

$$
\begin{aligned}
& S=\left\{x \in \mathbb{E}^{a} \mid \forall y \in \mathbb{E}^{a}(f(x)=f(y)) \wedge \forall \varepsilon_{1}>0, \exists \varepsilon_{2}>0 \wedge \delta_{1}>0 \wedge \delta_{2}>0,\right. \\
&\left.f\left(U_{y}\left(\delta_{1}\right)\right) \subseteq f\left(U_{x}\left(\varepsilon_{2}\right)\right) \subseteq f\left(U_{y}\left(\delta_{2}\right)\right) \subseteq f\left(U_{x}\left(\varepsilon_{1}\right)\right)\right\} .
\end{aligned}
$$

By the basic results of elimination theory, especially Theorem 3.3.15 and Corollary 3.319 of [Ma] (see [Se, Theorem 3] and [BR, Theorem 2.3.4] for some historical precedents of this Tarski-Seidenberg theory, also) we know that $S$ is an integral semi-algebraic set. Since $\mathbf{p}$ is a generic point in $\mathbb{E}^{a}$ and it is not in the set $T$, we know that it must be in the topological interior of $S$, since if one of the set inclusions in the definition of $S$ does not hold for $\mathbf{p}$, then $f(\mathbf{p})$ will lie in the integral algebraic set defined by the intersection of those images $f\left(U_{\mathbf{p}}\right) \cap f\left(U_{\mathbf{q}}\right)$ and be of dimension less than $m$. Then $f^{-1}\left(f\left(U_{\mathbf{p}} \cap f\left(U_{\mathbf{q}}\right)\right)\right)$ will be an integral algebraic set of dimension less than $a$. This contradicts $\mathbf{p}$ being generic.

So there is a neighborhood of $f(\mathbf{p})=f(\mathbf{q})$ in $f\left(\mathbb{E}^{a}\right)$ diffeomorphic to $\mathbb{E}^{m}$. However, there is more information. Let $\hat{h}: \mathbb{E}^{m} \rightarrow f\left(\mathbb{E}^{a}\right) \subset \mathbb{E}^{b}$ be such a diffeomorphism, and let $h: \mathbb{E}^{m} \times \mathbb{E}^{a-m} \rightarrow f\left(\mathbb{E}^{a}\right) \subset \mathbb{E}^{b}$ be $\hat{h}$ preceeded by projection onto the first factor. The local submersion theorem (see page 20 of [GP]) implies that there are diffeomorphisms $h_{\mathbf{p}}: N_{\mathbf{p}} \rightarrow \mathbb{E}^{m} \times \mathbb{E}^{a-m}$ and $h_{\mathbf{q}}: N_{\mathbf{q}} \rightarrow \mathbb{E}^{m} \times \mathbb{E}^{a-m}$ such that $h h_{\mathbf{p}}=f$ and $h h_{\mathbf{q}}=f$, where $N_{\mathbf{p}}$ is a neighborhood of $\mathbf{p}$ in $\mathbb{E}^{a}$ and $N_{\mathbf{q}}$ is a neighborhood of $\mathbf{q}$ in $\mathbb{E}^{a}$. Then we define $g=h_{\mathbf{p}}^{-1} h_{\mathbf{q}}$. The following commutative diagram sums up the above argument:

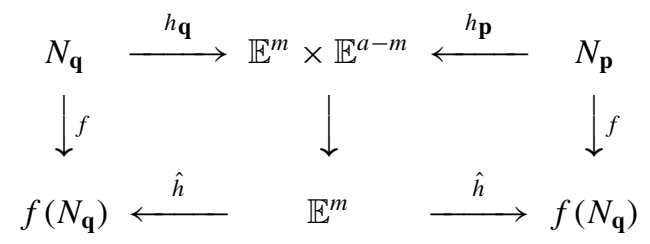

\section{The Stress Matrix and Affine Maps}

We are now ready to apply Proposition 3.3 to stresses. The following is a weak version of Theorem 1.3.

Theorem 4.1. Suppose that $\mathbf{p}=\left(p_{1}, \ldots, p_{n}\right)$ is a generic configuration in $\mathbb{E}^{d}$ such that there is a non-zero equilibrium stress $\omega$ for a framework $G(\mathbf{p})$, where the rank of the associated stress matrix $\Omega$ is $n-d-1$, and $G(\mathbf{p}) \equiv G(\mathbf{q})$. Then $\mathbf{q}$ is an affine image of $\mathbf{p}$.

Proof. Apply Proposition 3.3 to the rigidity map $f: \mathbb{E}^{n d} \rightarrow \mathbb{E}^{e}$ to get a diffeomorphism $g: N_{\mathbf{q}} \rightarrow N_{\mathbf{p}}$ from a neighborhood of $\mathbf{q}$ to a neighborhood of $\mathbf{p}$ such that $f g=f$ and $g(\mathbf{q})=\mathbf{p}$. Taking differentials we get $d f_{\mathbf{q}}=d f_{\mathbf{p}} d g_{\mathbf{q}}$, where $d g_{\mathbf{q}}$ is non-singular. Rewriting this in terms of rigidity matrices, we get $R(\mathbf{q})=R(\mathbf{p}) d g_{\mathbf{q}}$. Thus $\omega R(\mathbf{q})=$ $\omega R(\mathbf{p}) d g_{\mathbf{q}}=0$. In other words, $G(\mathbf{p})$ and $G(\mathbf{q})$ have the same space of equilibrium stresses. By Proposition 1.2(ii) $\mathbf{q}$ is an affine image of $\mathbf{p}$. 
Next we have to deal with the possibility that there may be non-congruent affine images $\mathbf{q}$ of $\mathbf{p}$ such that $G(\mathbf{p}) \equiv G(\mathbf{q})$. We recall some basic properties of affine maps and quadratic forms.

Let $Q$ be a symmetric $d$-by- $d$ matrix. It defines a conic at infinity by $C(Q)=$ $\left\{p \in \mathbb{E}^{d} \mid p^{\mathrm{T}} Q p=0\right\}$. (This is a cone and can be thought of as a "pre-homogeneous" description of a conic in projective $(d-1)$-dimensional space $\mathbb{R} \mathbb{P}^{d-1}$.) If a framework $G(\mathbf{p})$ is in $\mathbb{E}^{d}$, we say its edge directions are on a conic at infinity if there is a non-zero symmetric $d$-by- $d$ matrix $Q$ such that for all $\{i, j\}$ edges of $G, p_{i}-p_{j} \in C(Q)$. The following can be found in [C3, Theorem 5.5], but we provide a quick proof here.

Proposition 4.2. Suppose that $\omega$ is an equilibrium stress for a framework $G(\mathbf{p})$ in $\mathbb{E}^{d}$ such that $\omega_{i j} \neq 0$ for all $\{i, j\}$ edges of $G$. Every affine map of $\mathbf{p}$ preserving edge lengths of $G(\mathbf{p})$ is a congruence if and only if the edge directions of $G(\mathbf{p})$ do not lie on a conic at infinity.

Proof. An affine map defined on $\mathbb{E}^{d}$ is given by $p \rightarrow A p+r$, where $A$ is a $d$-by- $d$ matrix and $r \in \mathbb{E}^{d}$ is constant. Such a map preserves the edge lengths of $G(\mathbf{p})$ if and only if for all $\{i, j\}$ edges of $G$,

$$
\begin{aligned}
\left|p_{i}-p_{j}\right|^{2} & =\left(p_{i}-p_{j}\right)^{\mathrm{T}}\left(p_{i}-p_{j}\right)=\left(A p_{i}-A p_{j}\right)^{\mathrm{T}}\left(A p_{i}-A p_{j}\right) \\
& =\left(p_{i}-p_{j}\right)^{\mathrm{T}} A^{\mathrm{T}} A\left(p_{i}-p_{j}\right),
\end{aligned}
$$

where we regard vectors as column matices and ()$^{\mathrm{T}}$ is the transpose operation. Define $Q=I-A^{\mathrm{T}} A$. Then the edge length corresponding to $\{i, j\}$ is preserved if and only if $\left(p_{i}-p_{j}\right)^{\mathrm{T}} Q\left(p_{i}-p_{j}\right)=0$. On the other hand, the affine map defined by $A$ is a congruence if and only if $Q=I-A^{\mathrm{T}} A=0$. In other words, $A$ is othogonal. Thus if the edge directions do not lie on a conic at infinity, then there cannot be any affine map that preserves the edge lengths of $G(\mathbf{p})$, other than a congruence.

Conversely, suppose that the edge directions do lie on a conic at infinity $C(Q)$, defined by the symmetric matrix $Q$. Then there is an $\varepsilon \neq 0$ such that $I-\varepsilon Q$ is positive definite, and we can find a matrix $A$ such that $A^{\mathrm{T}} A=I-\varepsilon Q$. By (6), $A$ provides the required affine map.

Generally, it is a nuisance to determine that there are no affine maps that are not congruences. In the plane the conic at infinity consists of at most two directions, i.e., at most two points on $\mathbb{R P}^{1}$, the real projective line. In $\mathbb{E}^{3}$ a conic at infinity is determined by five distinct points, no three collinear. In dimension $d$ a conic is determined by $d(d+1) / 2$ points. In the generic case we have the following:

Proposition 4.3. Suppose that $G(\mathbf{p})$ is a framework in $\mathbb{E}^{d}$ such that $G$ is a finite graph, each vertex of $G$ has degreee at least $d$, and $\mathbf{p}=\left(p_{1}, \ldots, p_{n}\right)$ is a generic configuration. Then the edge directions of $G(\mathbf{p})$ do not lie on a conic at infinity.

Proof. It is enough to find one configuation $\mathbf{p}$ in $\mathbb{E}^{d}$, not necessarily generic, such that the edge directions of $G(\mathbf{p})$ do not lie on a conic at infinity, because the linear equations 
$\left(p_{i}-p_{j}\right)^{\mathrm{T}} Q\left(p_{i}-p_{j}\right)=0$ that determine a matrix $Q$ depend continuously on the configuration $\mathbf{p}$. When these linear equations are inconsistent there is an arbitrarily small perturbation of a non-generic configuration to a generic one so the equations remain inconsistent.

We proceed by induction on the dimension $d$ of the ambient space, starting at $d=2$. Each vertex of $G$ has degree at least 2 , and so $n \geq 3$, and the conic in this dimension consists of two directions, which are taken up by the edges directions at any vertex. Then at least one of the other edge directions at any other vertex will be a third direction. For the general inductive step, remove a vertex, say the $n$th vertex of $G$, to get a new graph which has $n-1$ vertices, each of which has degree at least $d-1$. Place these $n-1$ vertices in a $(d-1)$-dimensional plane parallel to the first $d-1$ coordinate vectors, say $\mathbb{E}^{d-1}+k$, where $k \neq 0$ is a constant vector and $e_{1}, e_{2}, \ldots, e_{d-1}$ span $\mathbb{E}^{d-1}$. Then in $\mathbb{E}^{d-1}+k$, make sure the points of the configuration $\left(p_{1}, \ldots, p_{n-1}\right)$ are such that they do not lie on any conic at infinity, by induction. (For example, a configuration in $\mathbb{E}^{d-1}$, generic with respect to the first $d-1$ coordinates, translated by $k$, will have this property, if any configuration does.)

Suppose $Q$ is an $n$-by- $n$ matrix defining a conic at infinity $C(Q)$. Regarding $Q$ as a quadratic form, it is zero when restricted to $\mathbb{E}^{d-1}$, by the induction hypothesis. In other words, $x^{\mathrm{T}} Q x=0$ for all $x \in \mathbb{E}^{d-1}$. Let $\left(p_{1}, \ldots, p_{d}\right)$ be $d$ points of $\mathbf{p}$ that are adjacent to $p_{n}=0$ in $G$, and we may assume that these vectors are a basis for $\mathbb{E}^{d}$. So for $1 \leq i \leq d$, we have $\left(p_{i}-p_{n}\right)^{\mathrm{T}} Q\left(p_{i}-p_{n}\right)=p_{i}^{\mathrm{T}} Q p_{i}=0$. For $1 \leq i<j \leq d$, we get $\left(p_{i}-p_{j}\right)^{\mathrm{T}} Q\left(p_{i}-p_{j}\right)=p_{i}^{\mathrm{T}} Q p_{i}+p_{j}^{\mathrm{T}} Q p_{j}-2 p_{i}^{\mathrm{T}} Q p_{j}=-2 p_{i}^{\mathrm{T}} Q p_{j}=0$, because $p_{i}-p_{j} \in \mathbb{E}^{d-1}$. With respect to the basis $\left(p_{1}, \ldots, p_{d}\right)$, the matrix $Q$ is zero. Thus, for this configuration, the edges of $G(\mathbf{p})$ do not lie on a conic at infinity, and the same must hold for a generic configuration in $\mathbb{E}^{d}$.

\section{Proof of the Main Results}

We put the results together that we have at this point to prove the main theorem.

Proof of Theorem 1.3. Suppose that $\mathbf{p}=\left(p_{1}, \ldots, p_{n}\right)$ is a generic configuration in $\mathbb{E}^{d}$ and that $G(\mathbf{p})$ is a framework with a non-zero equilibrium stress $\omega$ whose stress matrix $\Omega$ has rank $n-d-1$. We can assume, without loss of generality, by restricting to a subgraph if necessary, that all of the edges $\{i, j\}$ of $G$ have $\omega_{i j} \neq 0$. (If there is more than one component of stressed edges of $G$, then the rank of $\Omega$ will be strictly less than $n-d-1$.) Let $\mathbf{q}$ be another configuration in $\mathbb{E}^{d}$ such that $G(\mathbf{p}) \equiv G(\mathbf{q})$. By Theorem 4.1, the configuration $\mathbf{q}$ is an affine image of $\mathbf{p}$. Since the configuration $\mathbf{p}$ is generic, no $d+1$ of the vertices lie in a $(d-1)$-dimensional affine subspace, and the affine span of the vertices of $\mathbf{p}$ is all of $\mathbb{E}^{d}$. Since each of the edges of $G(\mathbf{p})$ has a non-zero equilibrium stress, for any fixed vertex $i$, the vectors $p_{j}-p_{i}$, for $\{i, j\}$ an edge of $G$, must be dependent by the equilibrium equation (1). Thus the degree of any vertex of $G$ is at least $d+1>d$. So we can apply Proposition 4.3 to conclude that the edge directions of $G(\mathbf{p})$ do not lie on a conic at infinity in $\mathbb{E}^{d}$. Then Proposition 4.2 implies that $\mathbf{p} \equiv \mathbf{q}$, as desired. 
Next we wish to apply the above results to prove Theorem 1.5, which is the one of the main ingredients for characterizing generic global rigidity in the plane. We say that the distance between linear subspaces $L_{1}$ and $L_{2}$ of $\mathbb{E}^{n}$ is the Hausdorf distance between $L_{1} \cap \mathbb{S}^{n-1}$ and $L_{2} \cap \mathbb{S}^{n-1}$, where $\mathbb{S}^{n-1}$ is the unit sphere in $\mathbb{E}^{n}$. First a lemma.

Lemma 5.1. Suppose that $A\left(x_{1}, \ldots, x_{n}\right)$ is a matrix whose entries are integral polynomial functions of the real variables $x=\left(x_{1}, \ldots, x_{n}\right)$. Let $m$ be the maximum rank of $A(x)$, and for $x=\bar{x}$ suppose that the rank of $A(\bar{x})=m$. Then for any $\varepsilon>0$ there is a $\delta>0$ such that for $|x-\bar{x}|<\delta$, the distance between $\operatorname{ker} A(x)$ and $\operatorname{ker} A(\bar{x})$ is less than $\varepsilon$.

Proof. The equations that define the kernel of $A(x)$ are rational functions that are defined as long as the determinants of some $m$-by- $m$ minor is non-zero. Thus ker $A(x) \cap$ $\mathbb{S}^{n-1}$ varies continuously and the conclusion follows.

We consider the Hennenberg operation on a framework in $\mathbb{E}^{d}$. Recall that this subdivides an edge of $G$ at a point and adds $d-1$ edges from that vertex to $d-1$ other vertices, not the endpoints of the original edge, to obtain a new framework $\sigma G(\sigma \mathbf{p})$. We now show how to prove the main result:

Proof of Theorem 1.5. Suppose that $\{i, j\}$ is the edge of $G$ to be subdivided, and let $p_{n+1}=\left(p_{i}+p_{j}\right) / 2$ be the new vertex on the line through $p_{i}$ and $p_{j}$. Let $\omega_{i j}$ be the stress corresponding to the edge $\{i, j\}$ of $G$, coming from the equilibrium stress $\omega=\left(\ldots, \omega_{i j}, \ldots\right)$ for $G(\mathbf{p})$. Remove $\{i, j\}$ and replace $\omega_{i j}$ with the two stresses $\omega_{i, n+1}=$ $\omega_{j, n+1}=2 \omega_{i, j}$. Call this new stress $\sigma \omega$, the new configuration $\sigma \mathbf{p}=\left(p_{1}, \ldots, p_{n}, p_{n+1}\right)$, and the new graph $\sigma G$, where in addition to the edges $\{i, n+1\}$ and $\{j, n+1\}$, there are $d-1$ edges from $n+1$ to $d-1$ distinct vertices other than $i$ and $j$, where these $d-1$ edges have zero stress.

By checking the equilibrium condition at $i, j$, we see that $\sigma \omega$ is an equilibrium stress for $\sigma G(\sigma \mathbf{p})$. Note that the rank condition (i) is equivalent to the configuration $\mathbf{p}$ being universal with respect to $\omega$. In other words, the dimension of the affine span of $\mathbf{p}$ is maximum given that $\omega$ is an equilibrium stress by condition (ii).

We claim that the configuration $\sigma \mathbf{p}$ is universal for $\sigma \omega$ as well. If not, there is another configuraton $\tilde{\mathbf{p}}$, which is universal for $\sigma \omega$ in dimension $\tilde{d}>d$. However, we can reverse the subdivision process and eliminate the vertex $\tilde{p}_{n+1}$ as well as the additional edges from $n+1$, which have zero stress anyway. Nonetheless, then the affine span of $\left(\tilde{p}_{1}, \ldots, \tilde{p}_{n}\right)$ is still $\tilde{d}>d$, since $\tilde{p}_{n+1}$ lies on the line segment between $\tilde{p}_{i}$ and $\tilde{p}_{j}$. This contradicts the assumption that $G(\mathbf{p})$ was universal. So the rank of the associated stress matrix $\Omega$ for $\sigma G(\sigma \mathbf{p})$ is $n+1-d-1=n-d$. However, $\sigma \mathbf{p}$ is not generic in $\mathbb{E}^{d}$.

Since $G(\mathbf{p})$ is infinitesimally rigid, then Proposition 2.3 implies that $\sigma G(\sigma \mathbf{p})$ is also infinitesimally rigid. By Lemma 5.1 the coordinates of the stress and therefore the entries in the stress matrix itself are only perturbed by a small amount when $\sigma G(\sigma \mathbf{p})$ is perturbed to $\sigma G(\mathbf{q})$, where $\mathbf{q}$ is generic. Thus $\mathbf{q}$ is still is universal with respect a stress on $G(\mathbf{q})$. This shows condition (i). 
The set of infinitesimally rigid configurations is open in the space of all configurations, so condition (ii) holds as well for $\sigma G(\mathbf{q})$.

\section{History}

One of the motivations for this work was the "Molecule Problem." Suppose that a framework $G(\mathbf{p})$ in $\mathbb{E}^{d}$ exists, but we are given only the edge lengths. Find a corresponding configuration $\mathbf{q}$ in $\mathbb{E}^{d}$ such that $G(\mathbf{q})$ has the same edge lengths as $G(\mathbf{p})$. In [H1] there is an algorithm proposed to solve this problem, particularly in $\mathbb{E}^{2}$. Roughly, the idea is to use a divide-and-conquer algorithm, and it depends on breaking up $G$ into globally rigid pieces and fitting them together. (The terminology there was "uniquely rigid" instead of global rigidity.) This led to Theorem 1.1 of Hendrickson, which in the plane did not lead to any known examples of graphs that were not globally rigid. This, in turn, led to the combinatorial conjecture for graphs in the plane with $2 n-2$ edges that was solved in [BJ]. Flushed with that success, the main result of [JJ] went on to solve the more general case with any number of edges, Theorem 1.6 here.

Theorem 1.3 was cited in Chapter 7 of [H1] without proof, as a sufficient condition for generic global rigidity in any dimension. The example of $K(5,5)$ in $\mathbb{E}^{3}$ was also mentioned in [H1] and appeared in [C2].

\section{Acknowledgments}

The author thanks Tibor Jordán, Maria Sloughter, Walter Whiteley, and both referees for several very useful comments and corrections.

\section{References}

[AR1] Asimow, Len; Roth, Ben. The rigidity of graphs. Trans. Amer. Math. Soc. 245 (1978), 279-289. MR 80i:57004a.

[AR2] Asimow, Len; Roth, Ben. The rigidity of graphs, II. J. Math. Anal. Appl. 68(1) (1979), 171-190. MR 80i:57004b.

[BJ] Berg, Alex R.; Jordán, Tibor. A proof of Connelly's conjecture on 3-connected circuits of the rigidity matroid. J. Combin. Theory Ser. B, $\mathbf{8 8}$ (2003) 77-97.

[BR] Benedetti, Riccardo; Risler, Jean-Jacques. Real algebraic and semi-algebraic sets. Actualités Mathématiques. [Current Mathematical Topics] Hermann, Paris, 1990, 340 pp. ISBN: 2-7056-6144-1. MR 99a:57012.

[C1] Connelly, Robert. Rigidity and energy. Invent. Math. 66(1) (1982), 11-33. MR 83m:52012.

[C2] Connelly, Robert. Generic global rigidity. Applied Geometry and Discrete Mathematics, pp. 147-155; DIMACS Series in Discrete Mathematics and Theoretical Computer Science, 4. American Mathematical Society, Providence, RI, 1991. MR 83m:52012.

[C3] Connelly, Robert. Rigidity. Handbook of Convex Geometry, Vols. A, B, pp. 223-271. North-Holland, Amsterdam, 1993. MR 94j:52041.

[CW] Connelly, Robert; Whiteley, Walter. Second-order rigidity and prestress stability for tensegrity frameworks. SIAM J. Discrete Math. 9(3) (1996), 453-491. MR 97e:52037.

[G] Gluck, Herman. Almost all simply connected closed surfaces are rigid. Geometric Topology (Proc. Conf., Park City, Utah, 1974), pp. 225-239. Lecture Notes in Mathematics, Vol. 438, Springer-Verlag, Berlin, 1975. MR 53 \#4074. 
[GP] Guillemin, Victor; Pollack, Alan. Differential Topology. Prentice-Hall, Inc., Englewood Cliffs, NJ, 1974. MR 50 \#1276.

[GSS] Graver, Jack; Servatius, Brigitte; Servatius, Herman. Combinatorial Rigidity. Graduate Studies in Mathematics, 2. American Mathematical Society, Providence, RI, 1993. MR 95b:52034.

[H1] Hendrickson, Bruce. The Molecule Problem: Determining Conformation from Pairwise Distances. Ph.D dissertation, Cornell University, 1991.

[H2] Hendrickson, Bruce. Conditions for unique graph realizations. SIAM J. Comput. 21(1) (1992), 65-84. MR 92m:05182.

[H3] Hendrickson, Bruce. The molecule problem: exploiting structure in global optimization. SIAM J. Optim. 5(4) (1995), 835-857. MR 96g:90093.

[JJ] Jackson, Bill; Jordán, Tibor. Connected rigidity matroids and unique realizations of graphs (preprint) (2003).

[Ma] Marker, David. Model Theory. An Introduction. Graduate Texts in Mathematics, 217. Springer-Verlag, New York, 2002.

[Mi] Milnor, John W. Topology from the Differentiable Viewpoint. Based on Notes by David W. Weaver. Revised reprint of the 1965 original. Princeton Landmarks in Mathematics. Princeton University Press, Princeton, NJ, 1997. MR 98h:57051.

[Sa] Saxe, James B. Embeddability of weighted graphs in $k$-space is strongly NP-hard. Technical report, Computer Science Department, Carnegie Mellon University, 1979.

[Se] Seidenberg, A. A new decision method for elementary algebra. Ann. of Math. (2) 60, (1954), 365-374. MR 16,209a.

[TW] Tay, Tiong-Seng (SGP-Sing); Whiteley, Walter. Generating isostatic frameworks. (Dual FrenchEnglish text.) Structural Topology 11 (1985), 21-69. MR 87e:05139.

Received October 28, 2003, and in revised form February 24, 2004. Online publication October 20, 2004. 